\title{
Investigation of the Interaction between Patulin and Human Serum Albumin by a Spectroscopic Method, Atomic Force Microscopy, and Molecular Modeling
}

\author{
Li Yuqin, ${ }^{1}$ You Guirong, ${ }^{1}$ Yang Zhen, ${ }^{2}$ Liu Caihong, ${ }^{1}$ Jia Baoxiu, ${ }^{1}$ \\ Chen Jiao, ${ }^{3}$ and Guo Yurong ${ }^{4}$ \\ ${ }^{1}$ College of Pharmacy, Taishan Medical University, Taian 271016, China \\ ${ }^{2}$ College of Clinic, Taishan Medical University, Taian 271016, China \\ ${ }^{3}$ College of Life Science and Technology, China Pharmaceutical University, Nanjing 210009, China \\ ${ }^{4}$ College of Food Engineering and Nutritional Science, Shaanxi Normal University, Xian 710062, China \\ Correspondence should be addressed to Li Yuqin; liyuqin@tsmc.edu.cn
}

Received 27 February 2014; Accepted 8 April 2014; Published 8 July 2014

Academic Editor: Kazuma Ogawa

Copyright (C) 2014 Li Yuqin et al. This is an open access article distributed under the Creative Commons Attribution License, which permits unrestricted use, distribution, and reproduction in any medium, provided the original work is properly cited.

\begin{abstract}
The interaction of patulin with human serum albumin (HSA) was studied in vitro under normal physiological conditions. The study was performed using fluorescence, ultraviolet-visible spectroscopy (UV-Vis), circular dichroism (CD), atomic force microscopy (AFM), and molecular modeling techniques. The quenching mechanism was investigated using the association constants, the number of binding sites, and basic thermodynamic parameters. A dynamic quenching mechanism occurred between HSA and patulin, and the binding constants $(K)$ were $2.60 \times 10^{4}, 4.59 \times 10^{4}$, and $7.01 \times 10^{4} \mathrm{M}^{-1}$ at 288,300 , and $310 \mathrm{~K}$, respectively. Based on fluorescence resonance energy transfer, the distance between the HSA and patulin was determined to be $2.847 \mathrm{~nm}$. The $\Delta G^{0}$, $\Delta H^{0}$, and $\Delta S^{0}$ values across various temperatures indicated that hydrophobic interaction was the predominant binding force. The UV-Vis and CD results confirmed that the secondary structure of HSA was altered in the presence of patulin. The AFM results revealed that the individual HSA molecule dimensions were larger after interaction with patulin. In addition, molecular modeling showed that the patulin-HSA complex was stabilized by hydrophobic and hydrogen bond forces. The study results suggested that a weak intermolecular interaction occurred between patulin and HSA. Overall, the results are potentially useful for elucidating the toxigenicity of patulin when it is combined with the biomolecular function effect, transmembrane transport, toxicological, testing and other experiments.
\end{abstract}

\section{Introduction}

Patulin is a toxic secondary metabolite produced by a variety of food spoilage fungi, particularly by Penicillium, Aspergillus genera, and Byssochlamys species [1]. Different foods, including fruits and grains, especially apple and its juice, can be affected by these fungi and become contaminated with patulin [2]. The highest patulin production has been observed at storage temperatures between 4 and $25^{\circ} \mathrm{C}$ [3-5]. Patulin has a strong affinity for sulfhydryl groups, inhibiting the activity of many enzymes $[6,7]$. It also has strong antibiotic properties [8] and was used to treat the common cold in the 1940s. Acute toxicity after high dosing in animals is expressed as agitation, convulsions, dyspnea, pulmonary congestion, edema, ulceration, hyperemia, and gastrointestinal tract distension [9-11]. Nausea, vomiting, gastrointestinal disturbance, and kidney damage have been reported in humans $[12,13]$. Patulin has been reported to be a genotoxic, reprotoxic, embryotoxic, and immunosuppressive compound [14-18]. To establish guidelines for human exposure to patulin, the JECFA lowered the provisional maximum tolerable daily intake (PMTDI) of patulin from 1 to $0.4 \mathrm{mg} / \mathrm{kg}$ body mass/day based on a noobserved-effect level (NOEL) of $43 \mathrm{mg} / \mathrm{kg}$ body mass/day and the use of a 100-fold safety factor [6].

Human serum albumin (HSA) containing 585 amino acid residues is the most abundant protein constituent of the 
circulatory system, contributing significantly to physiological functions as carrier proteins [19]. HSA contains three homologous $\alpha$-helical domains (I-III). Each of these domains is divided into $\mathrm{A}$ and $\mathrm{B}$ subdomains that contain six and four $\alpha$-helices, respectively [20,21]. Based solely on this sole structure, HSA has an ability to bind an unusually broad spectrum of ligands such as inorganic ions, various drugs, amino acids, and fatty acids [22]. Binding to HSA facilitates the transport of these ligands throughout the circulation [23]. Moreover, these bindings appear to affect the secondary and tertiary structure of albumin [24]. Without a doubt, the interaction of any toxicant with HSA affects the transport of nutrients and drugs. Recently, studies have been conducted on the binding of organic contaminants or toxins to HSA, for example, Arazine, ochratoxin A, methyl parathion, arsenic, perfluorooctanoic acid and phthalate esters, deoxynivalenol, and 2-mercaptobenzimidazole [25-32].

In this study, the interaction of patulin with HSA was investigated using steady-state and time-resolved fluorescence spectroscopy, UV-Vis spectroscopy, circular dichroism (CD) spectroscopy, atomic force microscopy (AFM), and molecular modeling.

\section{Materials and Methods}

2.1. Materials. HSA (fatty acid free $<0.05 \%$ ) was purchased from Sigma Chemical Company (USA), and patulin (Figure 1, analytical grade) was purchased from J \& $\mathrm{K}$ Technology Co., Ltd (USA). A $0.05 \mathrm{~mol} \cdot \mathrm{L}^{-1}$ Tris- $\mathrm{HCl}$ (pH 7.4) buffer solution was used to maintain the solution $\mathrm{pH}$. A $15 \mu \mathrm{mol} \cdot \mathrm{L}^{-1}$ HSA stock solution was prepared in Tris- $\mathrm{HCl}$ buffer solution and stored in the dark at $4^{\circ} \mathrm{C}$. Dilutions of the HSA stock with Tris-HCl buffer solution were prepared immediately before use. A $1.0 \mathrm{~mol} \cdot \mathrm{L}^{-1} \mathrm{NaCl}$ solution was used to maintain an ionic strength of $0.1 \mathrm{M}$. A $1.0 \mathrm{mmol} \cdot \mathrm{L}^{-1}$ patulin solution was prepared in double-distilled water. All other reagents were of analytical grade, and double-distilled water was used throughout all experiments.

\subsection{Apparatuses and Methods}

2.2.1. Steady-State and Synchronous Fluorescence Spectroscopy. Steady-state fluorescence spectra were obtained using an F4500 fluorescence spectrometer (Hitachi, Japan). The excitation and emission slit widths were $5 \mathrm{~nm}$. An excitation wavelength of $295 \mathrm{~nm}$ and emission wavelengths between 300 and $500 \mathrm{~nm}$ were recorded.

The interactions between patulin and HSA were measured by the fluorescence method. The appropriate concentrations of $\mathrm{HSA}, \mathrm{NaCl}$, and patulin $-15 \mu \mathrm{mol} \cdot \mathrm{L}^{-1}, 1.0 \mathrm{~mol} \cdot \mathrm{L}^{-1}$, and $1.0 \mathrm{mmol} \cdot \mathrm{L}^{-1}$, respectively - were added to a $10 \mathrm{~mL}$ volumetric flask; next, the mixture was diluted to graduation with Tris-HCl buffer solution. All experiments were performed at 288,300 , and $310 \mathrm{~K}$. The method of synchronous fluorescence spectroscopy was carried out as described above.

2.2.2. Time-Resolved Fluorescence Spectroscopy. Timeresolved fluorescence spectra were measured by steady-state spectroscopy (FLS-920) (Edinburgh Instruments) using

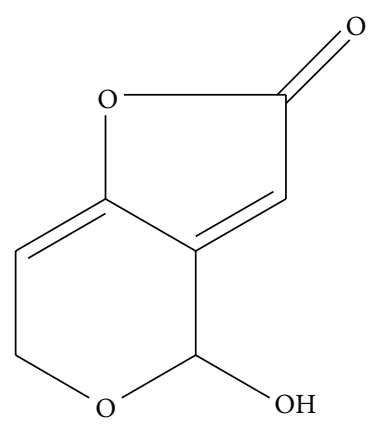

FIgURE 1: The chemical structure of patulin.

a standard time-correlated and single-photon counting scheme. Samples were excited with a subnanosecond pulsed diode laser at a repetition rate of $10 \mathrm{MHz}$ at $295 \mathrm{~nm}$. Fluorescence spectra were detected three times at $345 \mathrm{~nm}$ and at $288 \mathrm{~K}$. The decay of the fluorescence intensity was determined with the instrument-specific software based on tail fitting. The multiexponential values were assessed by considering the reduced chi-square value $\left(\chi^{2}<1.10\right)$ and the random distribution of the weighted residuals near zero.

2.2.3. UV-Vis Absorption Spectra. Absorption spectra were obtained with a UV-2450 UV-Vis spectrometer (Shimadzu, Japan) at $288 \mathrm{~K}$ in the range of $200-500 \mathrm{~nm}$ using a $1 \mathrm{~cm}$ quartz cell. The method employed was the same as that described in Section 2.2.1.

2.2.4. CD Spectra. CD measurements were collected with a JASCO J-810 circular dichroism spectrometer (Japan) at $288 \mathrm{~K}$ using a $0.1 \mathrm{~cm}$ quartz cell. The HSA concentration was $1.5 \mu \mathrm{mol} \cdot \mathrm{L}^{-1}$, and the scanning speed was $200 \mathrm{~nm} \mathrm{~min}{ }^{-1}$. The buffer solution was used as a blank and was automatically subtracted from the samples after scanning. Each sample was measured three times at a bandwidth of $1.0 \mathrm{~nm}$. The CD results were expressed in units of millidegrees.

2.2.5. Atomic Force Microscopy. AFM was carried out with a MultiMode Nanoscope IIIa (USA) equipped with a normal NP probe. The spring constant of the cantilever was $0.32 \mathrm{~N} / \mathrm{m}$, and the typical imaging resonance frequency of the fluid was $7-9 \mathrm{kHz}$. All of the samples were imaged in fluid contact mode with an O-ring liquid cell. Samples were prepared as follows: (1) free HSA with $30 \mu \mathrm{L}$ of $1.5 \mu \mathrm{mol} \cdot \mathrm{L}^{-1} \mathrm{HSA}$ was added to a mica substrate and incubated for $10 \mathrm{~min}$ at $288 \mathrm{~K}$ before washing with water; (2) HSA-patulin complexes with free HSA samples were prepared as described in step (1) prior to adding $20 \mu \mathrm{L}$ of a $15 \mu \mathrm{mol} \cdot \mathrm{L}^{-1}$ patulin solution, incubating for $15 \mathrm{~min}$, washing with water, drying under $\mathrm{N}_{2}$ for $4 \mathrm{~min}$, and imaging in air.

2.2.6. Molecular Docking. The 3D structure of the ligand was constructed with standard bond lengths and bond angles using the molecular modeling software program SYBYL8.0 (Tripos Inc., St. Louis, USA) for Linux. Geometry optimization was performed using the standard Tripos forcefield [33] 


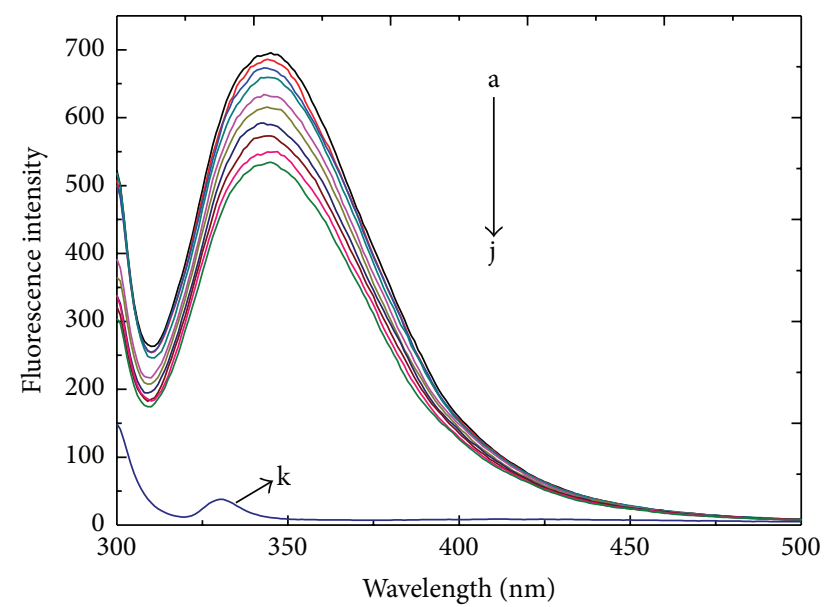

(a)

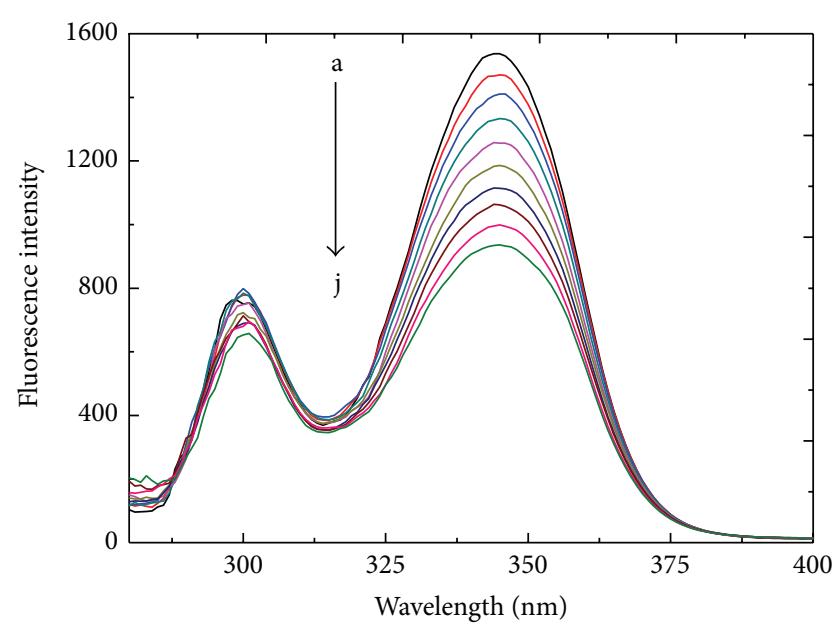

(b)

FIGURE 2: The fluorescence emission (a) and synchronous (b) spectra of the patulin-HSA system. The concentration of HSA was $1.5 \mu \mathrm{M}$, whereas the patulin concentrations were $0,3.33,6.67,10.0,13.3,16.7,20,23.3,26.7$, and $30.0 \mu \mathrm{M}$ from a to j, respectively. (k) [HAS] $=0 \mu \mathrm{M}$; [patulin] $=10.0 \mu \mathrm{M}$. Trisbuffer, $\mathrm{pH}=7.4, T=288 \mathrm{~K}$, and $\lambda_{\mathrm{ex}}=295 \mathrm{~nm}$.

with a distance-dependent dielectric function and an energy gradient of $0.001 \mathrm{kcal} / \mathrm{mol}$. Gasteiger-Hückel charges [34] were used.

Molecular docking was implemented using MOE2009 for Windows (Chemical Computing Group Inc., Montreal, Canada). The available X-ray structure of HSA complexed with R-warfarin (PDB code: 1H9Z) was applied in this work as the receptor. Hydrogen atoms were added to the PDB file. Then, the $1 \mathrm{H} 9 \mathrm{Z}$ complex was handled in LigX (a module of the MOE software) to meet the docking requirements. The conformer with the lowest $S$ value was used for further analysis.

\section{Results and Discussion}

3.1. Analysis of Fluorescence Quenching of HSA by Patulin. The intrinsic fluorescence of HSA is derived mainly from tryptophan (Trp), tyrosine (Tyr), and phenylalanine (Phe) residues. Phe residue fluorescence has a very low quantum yield, and Tyr residue fluorescence is nearly quenched when the residue is ionized or near an amino or carboxyl group of Trp. The only tryptophan residue (Trp-214) in HSA is located in domain II, Site I. The fluorescence of Trp-214 is sensitive to the ligand to which the residue binds. Therefore, Trp214 is often used as a probe to investigate the interaction of small ligands with HSA. Figure 2(a) shows the fluorescence spectra of HSA with different patulin concentrations at $\lambda_{\text {ex }}$ $295 \mathrm{~nm}$. The maximum emission wavelength $\left(\lambda_{\mathrm{em}}\right)$ of free HSA was $345 \mathrm{~nm}$. Its intensity gradually decreased with the concentration of patulin, and the peak position was slightly blue shifted. This result indicates that patulin could quench the intrinsic fluorescence of HSA and that Trp-214 was more polar and hydrophobic after the addition of patulin.

Synchronous fluorescence is a very useful tool for investigating the microenvironments of fluorophore functional groups. According to Miller [35], the fluorescence of HSA with a $\Delta \lambda\left(\Delta \lambda=\lambda_{\mathrm{em}}-\lambda_{\mathrm{ex}}\right)$ of $60 \mathrm{~nm}$ is characteristic of the alteration of the polarity of the microenvironment surrounding Trp-214 residues. The effect of patulin on synchronous fluorescence spectra is illustrated in Figure 2(b). The fluorescence intensity of Trp residues decreased with an increase in patulin concentration. The results correspond to those shown in the fluorescence spectra of Figure 2(a), suggesting that the microenvironment of Trp-214 was made more hydrophobic.

To further confirm the quenching mechanism, the fluorescence quenching data were analyzed with the SternVolmer equation:

$$
\frac{F_{0}}{F}=1+K_{\mathrm{SV}}[Q]
$$

where $F_{0}$ and $F$ are the fluorescence intensity in the absence and presence of a quencher, respectively, and $[Q]$ is the quencher concentration. $K_{\mathrm{SV}}$ is the Stern-Volmer quenching constant. The $K_{\mathrm{SV}}$ values at different temperatures are listed in Table 1. According to the fluorescence quenching mechanisms, a higher temperature would generally result in faster diffusion and the dissociation of weakly bound complexes [36]. The $K_{\mathrm{SV}}$ values increased with increasing temperature, which suggests that a dynamic quenching mechanism occurred between HSA and patulin.

Time-resolved fluorescence spectroscopy is a tool that can be used to investigate the interaction between ligands and proteins. The fluorescence lifetime can be used to directly differentiate between dynamic and static quenching. The lifetime of static quenching does not depend on the quencher concentration (i.e., $\left.\left\langle\tau_{0}\right\rangle=\langle\tau\rangle\right)[37,38]$. The timeresolved fluorescence spectra of the free HSA and patulinHSA complexes are shown in Figure 3, and the fluorescence decay parameters are listed in Table 2 . The fluorescence decay of the unquenched Trp-214 was biexponential $\left(\chi^{2}=1.007\right)$ (with $\tau_{1}=3.06 \mathrm{~ns}\left[\alpha_{1}\right.$ (fraction) $\left.=0.64\right], \tau_{2}=7.80 \mathrm{~ns}\left[\alpha_{2}\right.$ (fraction) $=0.36]$, and $\langle\tau\rangle=5.37 \mathrm{~ns})$. These results are in 
TABLE 1: Binding and thermodynamic parameters for the patulin-HSA interaction at different temperatures in Trisbuffer $(\mathrm{pH}=7.4)$.

\begin{tabular}{cccccccc}
\hline \multirow{2}{*}{$T / \mathrm{K}$} & \multicolumn{2}{c}{ Equation (2) } & \multicolumn{2}{c}{ Equation (3) } & $n$ & \multirow{2}{*}{$\Delta H^{0} / \mathrm{kJmol}^{-1}$} & $\Delta S^{0} / \mathrm{J} \mathrm{mol}^{-1}$ \\
& $K_{\mathrm{SV}} / \mathrm{M}^{-1}$ & $R$ & $K_{A} / \mathrm{M}^{-1}$ & $R$ & & $\Delta G^{0} / \mathrm{kJ} \mathrm{mol}^{-1}$ \\
\hline 288 & $1.17 \times 10^{4}$ & 0.9961 & $2.60 \times 10^{4}$ & 0.9769 & 1.10 & & -22.46 \\
300 & $1.40 \times 10^{4}$ & 0.9985 & $4.59 \times 10^{4}$ & 0.9914 & 1.17 & 8.06 & 106.0 \\
310 & $1.48 \times 10^{4}$ & 0.9972 & $7.01 \times 10^{4}$ & 0.9982 & 1.34 & & -23.73 \\
\hline
\end{tabular}

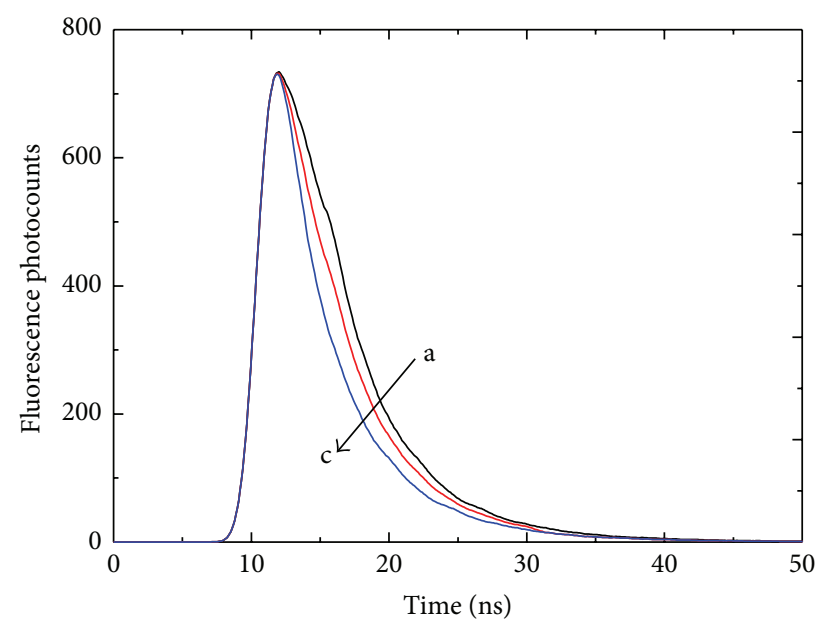

FIgURE 3: The time-resolved fluorescence decay of the patulin-HSA system. $C_{\mathrm{HSA}}=1.5 \mu \mathrm{M}$. Patulin concentrations were $0.0,1.5$, and $3.0 \mu \mathrm{M}$ from a to $\mathrm{c}$, respectively. Trisbuffer, $\mathrm{pH}=7.4, T=288 \mathrm{~K}$, and $\lambda_{\mathrm{ex}}=295 \mathrm{~nm}$.

agreement with those obtained for Trp-214 by Helms et al. [39]. As indicated in Table 2, the average fluorescence lifetime of Trp-214 decreased slightly with increasing patulin concentration. Therefore, these results suggest that the HSApatulin quenching mechanism was dynamic.

3.2. The Binding Constant and Binding Site. The apparent binding constant $\left(K_{A}\right)$ and the binding site number $(n)$ for the patulin-HSA complex were calculated by the following equation:

$$
\lg \frac{F_{0}-F}{F}=\lg K_{A}+n \lg [Q],
$$

where, $F_{0}$ and $F$ indicate the fluorescence intensity of the system in the absence and presence of a quencher and [Q] is the total quencher concentration. The values of $K_{A}$ and $n$ for patulin-HSA are also listed in Table 1 . The $K_{A}$ values increased with temperature, which indicates that the patulinHSA quenching mechanism was dynamic. This result agrees with the results described in Section 3.1.

3.3. Binding Mode. There are several types of noncovalent interaction modes between proteins and ligands, such as hydrogen bonds, van der Waals forces, hydrophobic interaction forces, and electrostatic forces [40]. The thermodynamic parameters, enthalpy change $\left(\Delta H^{0}\right)$, and entropy change $\left(\Delta S^{0}\right)$ of reaction are important for confirming what binding mode is active. Therefore, the temperature dependence of the binding constant was studied. $\Delta H^{0}$ and $\Delta S^{0}$ were obtained using the van't Hoff equations [(3) and (4)]:

$$
\ln K=-\frac{\Delta H^{0}}{R T}+\frac{\Delta S^{0}}{R}
$$

where $K$ is the binding constant obtained from (1) and $R$ is the universal gas constant. $\Delta H^{0}$ and $\Delta S^{0}$ were obtained from (4). Then, the free energy change $\left(\Delta G^{0}\right)$ was estimated using

$$
\Delta G^{0}=\Delta H^{0}-T \Delta S^{0} .
$$

The $\Delta H^{0}, \Delta S^{0}$, and $\Delta G^{0}$ values are also listed in Table 1 . The $\Delta H^{0}$ and $\Delta S^{0}$ values of patulin-HSA were $8.06 \mathrm{~kJ} \mathrm{~mol}^{-1}$ and $106.0 \mathrm{~J} \mathrm{~mol}^{-1} \mathrm{~K}^{-1}$, respectively. According to the results of Timasheff and Subramanian regarding various protein interactions $[41,42]$, a positive $\Delta S^{0}$ value is frequently regarded as evidence of a hydrophobic ligand-protein interaction considering the theory of the structure of water molecules. Thus, the hydrophobic interaction played a major role in the binding of patulin and HSA.

3.4. Energy Transfer between HSA and Patulin. The molecular distance $r$ between HSA and patulin can be determined using the Förster energy transfer theory. The overlap of patulin's UV absorption spectrum with its fluorescence spectrum is shown in Figure 4. Based on the Förster energy transfer theory, the distance $r$ can be calculated using

$$
E=1-\frac{F}{F_{0}}=\frac{R_{0}^{6}}{\left(R_{0}^{6}+r^{6}\right)},
$$

where $E$ is the efficiency of the transfer between HSA and patulin. $F$ and $F_{0}$ are the fluorescence intensities of HSA before and after the addition of patulin, respectively. $r$ is the distance between patulin and HSA, and $R_{0}$ is the critical distance at which the transfer efficiency is 50\% [30]. The value of $R_{0}$ is calculated using

$$
R_{0}^{6}=8.8 \times 10^{-25} K^{2} n^{-4} \phi J,
$$

where $K^{2}$ is the spatial orientation factor of the dipole, $n$ is the refractive index of the medium, and $\phi$ is the fluorescence quantum yield of HSA in the absence of an acceptor. In addition, $J$ is the overlap integral of the HSA and patulin fluorescence spectra. The value of $J$ is calculated using

$$
J=\frac{\sum F(\lambda) \varepsilon(\lambda) \lambda^{4} \Delta \lambda}{\sum F(\lambda) \Delta \lambda},
$$


TABLE 2: Fluorescence decay fitting parameters for the patulin-HSA system in Trisbuffer $(\mathrm{pH}=7.4)$.

\begin{tabular}{lcccccc}
\hline Substance & $\tau_{1}(\mathrm{~ns})$ & $\alpha_{1}$ & $\tau_{2}(\mathrm{~ns})$ & $\alpha_{2}$ & $\chi^{2}$ & $\tau$ (ns) \\
\hline HSA & 3.06 & 0.64 & 7.80 & 0.36 & 5.37 \\
Patulin-HAS (1:1) & 2.82 & 0.51 & 7.26 & 0.49 & 1.007 & 5.28 \\
Patulin-HAS (2:1) & 2.55 & 0.47 & 6.19 & 0.53 & 1.024 & 5.11 \\
\hline
\end{tabular}

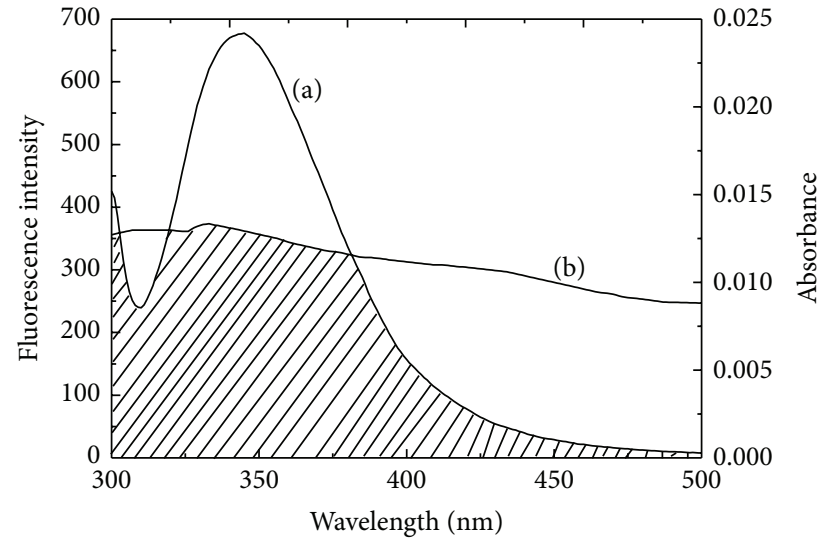

Figure 4: The overlap of (a) the absorption spectra of patulin and (b) the fluorescence emission spectrum of HSA. $C_{\mathrm{HSA}}=1.5 \mu \mathrm{M}$ and $C_{\text {patulin }}=1.5 \mu \mathrm{M}\left(288 \mathrm{~K}, \mathrm{pH}=7.4\right.$, and $\left.\lambda_{\text {ex }}=295 \mathrm{~nm}\right)$.

where $F(\lambda)$ denotes the fluorescence intensity of either donor at wavelength $\lambda$, and $\varepsilon(\lambda)$ denotesthe molar absorption coefficient of patulin at wavelength $\lambda\left(\mathrm{cm} \mathrm{mol}^{-1}\right)$. In this study, the value of $J$ was $1.896 \times 10^{-15} \cdot \mathrm{cm}^{3} \cdot \mathrm{L} \cdot \mathrm{mol}^{-1}$ and the value of $E$ was 0.0803 . For HSA, the $K^{2}$ is equal to $2 / 3$ and the $\phi$ is equal to 0.118 for an $n$ value of 1.336 . Thus, $R_{0}$ was $1.896 \mathrm{~nm}$, and $r$ was equal to $2.847 \mathrm{~nm}$. Clearly, the distance between HSA and patulin was less than $8 \mathrm{~nm}$ and within $0.5 R_{0}<r<1.5 R_{0}$. This result is in accordance with the Förster energy transfer theory and indicates that energy transfer occurred between patulin and HSA.

\subsection{Effect of Patulin on the Secondary Structure of HSA}

3.5.1. UV-Vis Results. Absorption at approximately $210 \mathrm{~nm}$ is indicative of the a-helix structure of HSA. Figure 5 shows the UV absorption spectra of HSA in the absence and presence of patulin. Figure 5 shows that HSA exhibited strong absorbance at $209 \mathrm{~nm}$ and weak absorbance at $280 \mathrm{~nm}$. The intensities of these two absorbance peaks increased with the addition of patulin, and there was little shift wavelengths. These two results clearly indicate that there was an interaction between patulin and HSA.

3.5.2. CD Results. CD spectroscopy is a quantitative technique that is used to study the conformation of proteins in aqueous solutions. The CD spectra of HSA exhibited two negative bands at 208 and $219 \mathrm{~nm}$, which characterize the $\alpha$ helical structure of the protein [23]. The CD spectra of the free HSA and HSA-patulin complexes are shown in Figure 6. The secondary structure of HSA was calculated using SELCON3

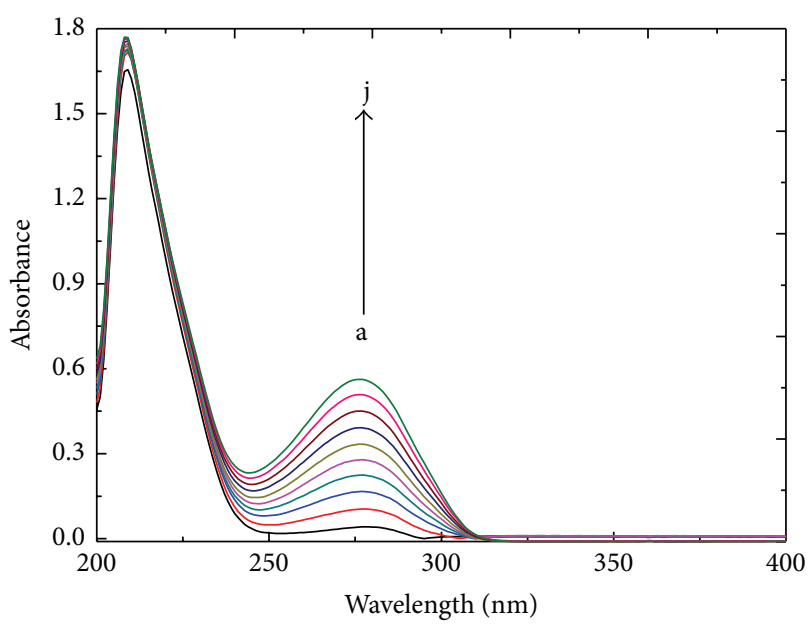

FIGURE 5: UV absorption spectra of the patulin-HSA system. The concentration of HSA was $1.5 \mu \mathrm{M}$, whereas the patulin concentrations were $0,3.33,6.67,10.0,13.3,16.7,20,23.3,26.7$, and $30.0 \mu \mathrm{M}$ from a to j, respectively. Trisbuffer, $\mathrm{pH}=7.4$, and $T=288 \mathrm{~K}$.

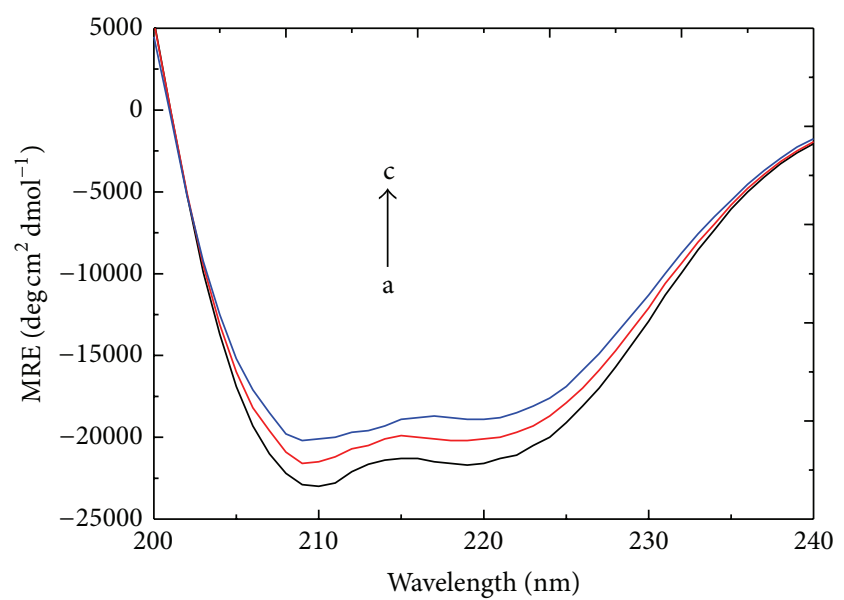

FIgure 6: The CD spectra of the patulin-HSA system. Patulin concentrations were $0.0,1.5$, and $3.0 \mu \mathrm{M}$ from a to $\mathrm{c}$, respectively. $C_{\mathrm{HSA}}=1.5 \mu \mathrm{M}, \mathrm{pH}=7.4$, and $T=288 \mathrm{~K}$.

(http://dichroweb.cryst.bbk.ac.uk/html); the results are listed in Table 3.

Table 3 shows that the free HSA had a high $\alpha$-helix content of $55.3 \%$ and contained $8.7 \% \beta$-sheets, $15.3 \% \beta$-turns, and $20.7 \%$ random coils. With patulin addition, the band intensity increased at all wavelengths and no significant peak shifts were observed; furthermore, the $\alpha$-helix content of HSA decreased to $50.7 \%$, accompanied by an increase in the 


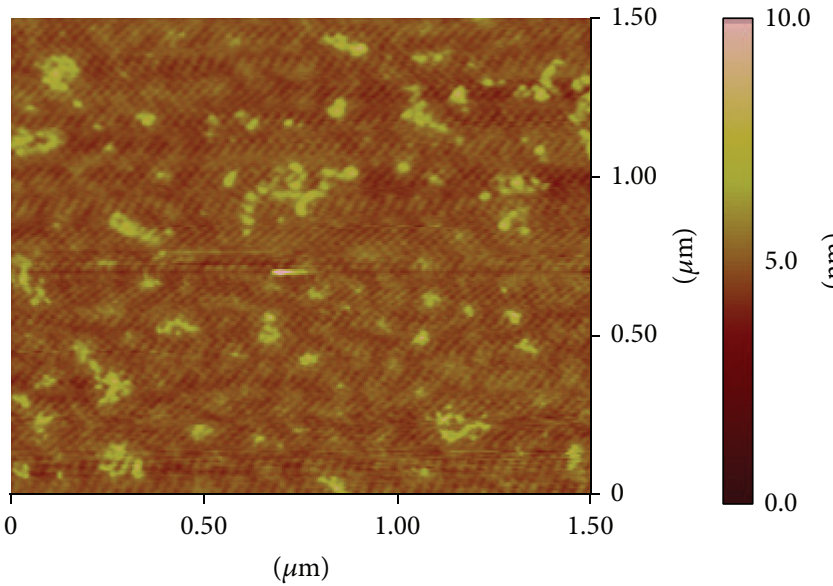

(a)

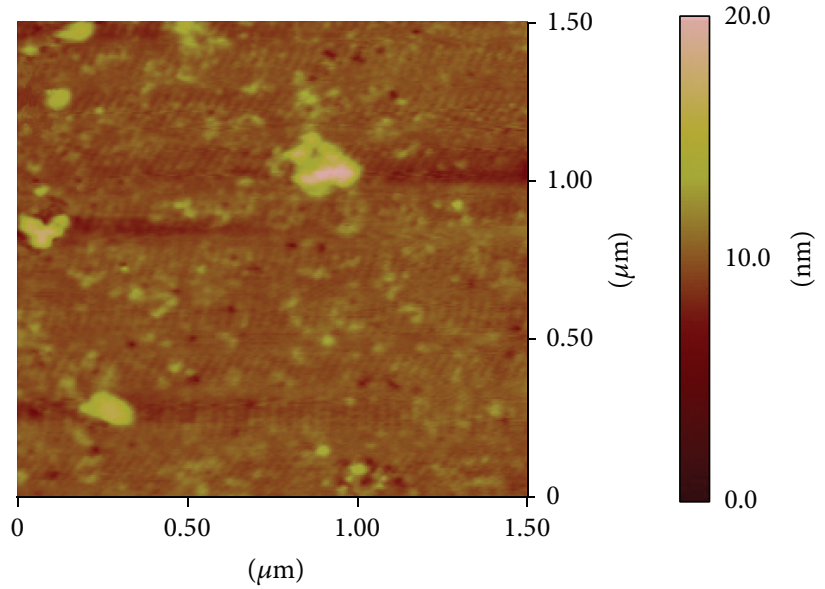

(b)

Figure 7: (a) An AFM topography image of free HSA and (b) an AFM topography image of the HSA-patulin complex. Samples were adsorbed onto mica under tapping mode in a Tris- $\mathrm{HCl}$ buffer solution, and the scan size of the image is $1.5 \mu \mathrm{m} \times 1.5 \mu \mathrm{m}$.

TABLE 3: Secondary structure determination for free HSA and its drug complexes in Trisbuffer $(\mathrm{pH}=7.4)$ at different molar concentration ratios for HSA and patulin.

\begin{tabular}{lcccc}
\hline Molar ratio (patulin-HSA) & $\alpha$-helical (\%) & $\beta$-sheet (\%) & $\beta$-turn (\%) & Random coil (\%) \\
\hline $0: 1$ & 55.3 & 8.7 & 15.3 & 20.7 \\
$1: 1$ & 52.7 & 9.1 & 15.6 & 22.6 \\
$2: 1$ & 50.7 & 10.6 & 17.4 & 21.3 \\
\hline
\end{tabular}

contents of $\beta$-sheets and $\beta$-turns to $10.6 \%$ and $17.4 \%$. The content of random coils was only slightly altered when the molar concentration ratio of patulin to HSA was increased from $0: 1$ to $2: 1$. Thus, the binding of HSA with patulin caused conformational changes in HSA and the loss of $\alpha$-helix stability.

3.5.3. The Interactions between Patulin and HSA Based on Atomic Force Microscopy. To study the changes in HSA topography with the addition of patulin, the free HSA and HAS-patulin complexes were imaged by AFM in triplicate. Figure 7 shows the AFM results obtained for the free HSA and its complexes.

As shown in Figure 7(a), HSA was adsorbed evenly on the mica surfaces. The mean height of the individual HSA molecules was $5.00 \pm 2.47 \mathrm{~nm}$. These dimensions are consistent with the results of previous AFM studies [43]. After the addition of patulin, the HSA molecule became swollen, and the mean height of HSA reached $15.2 \pm 3.05 \mathrm{~nm}$ (Figure 7(b)). The aggregation of the HSA molecules is also shown in Figure 7(b). The microenvironment around the HSA became more hydrophobic after interacting with patulin. To minimize any factors adversely affecting the formation of a stable structure, the HSA molecule reduced its surface area of contact with water by molecular aggregation. These results reveal that a hydrophobic interaction between HSA and patulin may occur.

3.6. Molecular Modeling. Crystal structure analyses indicated that HSA contains the three following domains, which are structurally similar (I-III): I (residues 1-195), II (196-383), and III (384-585). The principal ligand binding sites on HSA are located in the hydrophobic cavities in subdomains IIA and IIIA, which correspond to sites I and II (according to the terminology of Sudlow et al.) [44]. Site I of HSA, which is a preferred binding pocket within the IIA subdomain [44, 45], shows affinity for warfarin, phenylbutazone, and other drugs. In addition, site II shows affinity for ibuprofen, flufenamic acid, and other ligands. To obtain more information about the patulin-HSA binding sites, molecular docking was used to simulate the patulin-HSA interaction. Figure 8 shows the patulin-HSA interaction mode. Figure $8(\mathrm{~b})$ is a projection of Figure 8(a).

As shown in Figure 8, the patulin molecule was located within the binding pocket of site I (IIA), and the two rings were coplanar. The patulin molecule was inserted in the hydrophobic cavity that was formed by Ala291, Leu238, and Leu219. In addition, the pyran ring oxygen and 4-hydroxyl formed two hydrogen bonds with Arg222, and a furan ring carbonyl oxygen formed a hydrogen bond with His242. The docking results indicate that the interaction between patulin and HSA was dominated by hydrophobic forces accompanying with hydrogen bonds. In addition, several polar residues were observed near the patulin molecule, which played a subordinate role in stabilizing the ligand molecule through electrostatic interactions. For patulin-HSA, the calculated binding energy $\Delta G^{0}$ was $-26.68 \mathrm{~kJ} \mathrm{~mol}^{-1}$, which is near the experimental value $\left[-22.46 \mathrm{~kJ} \mathrm{~mol}^{-1}\right.$ at $\left.288 \mathrm{~K}\right]$, which further conformed the experimental results and demonstrated that the spontaneous binding between patulin and HSA occurred. 


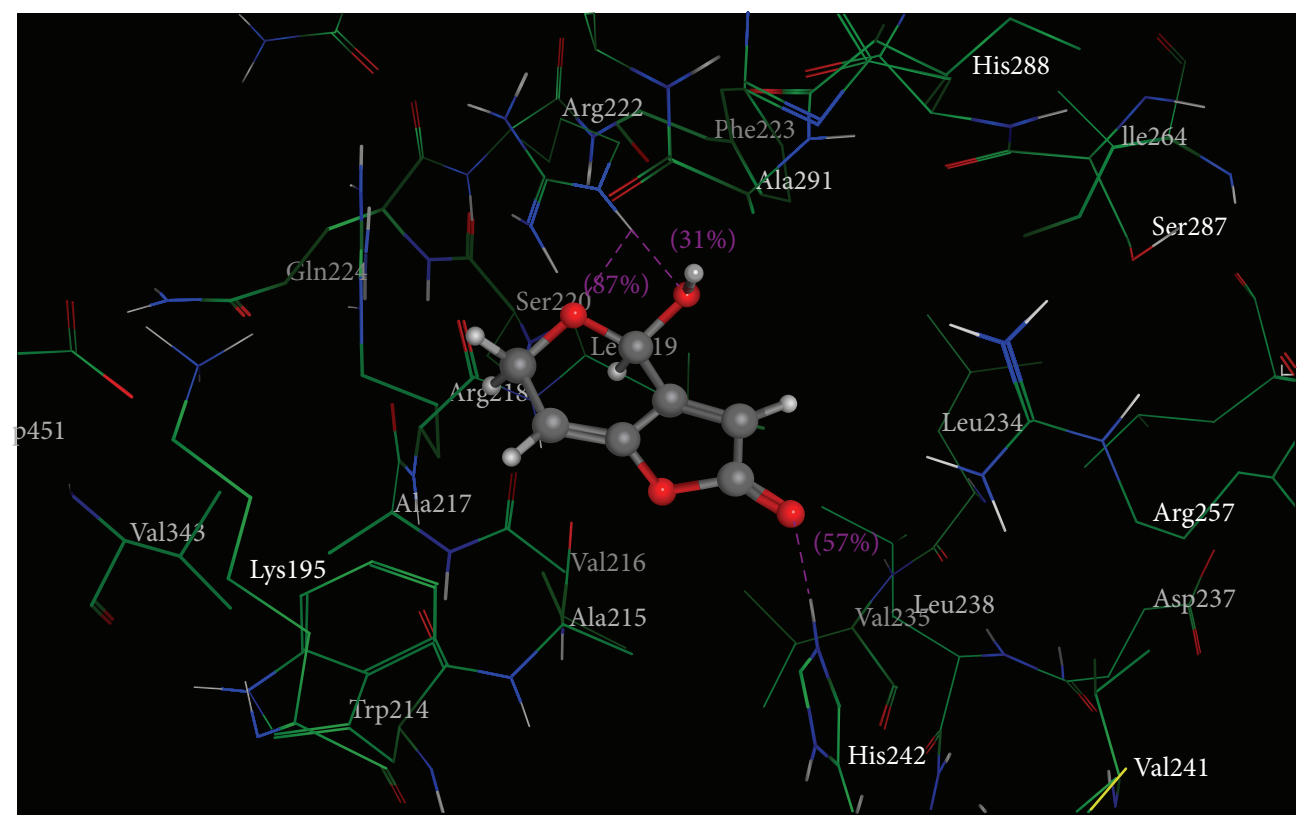

(a)
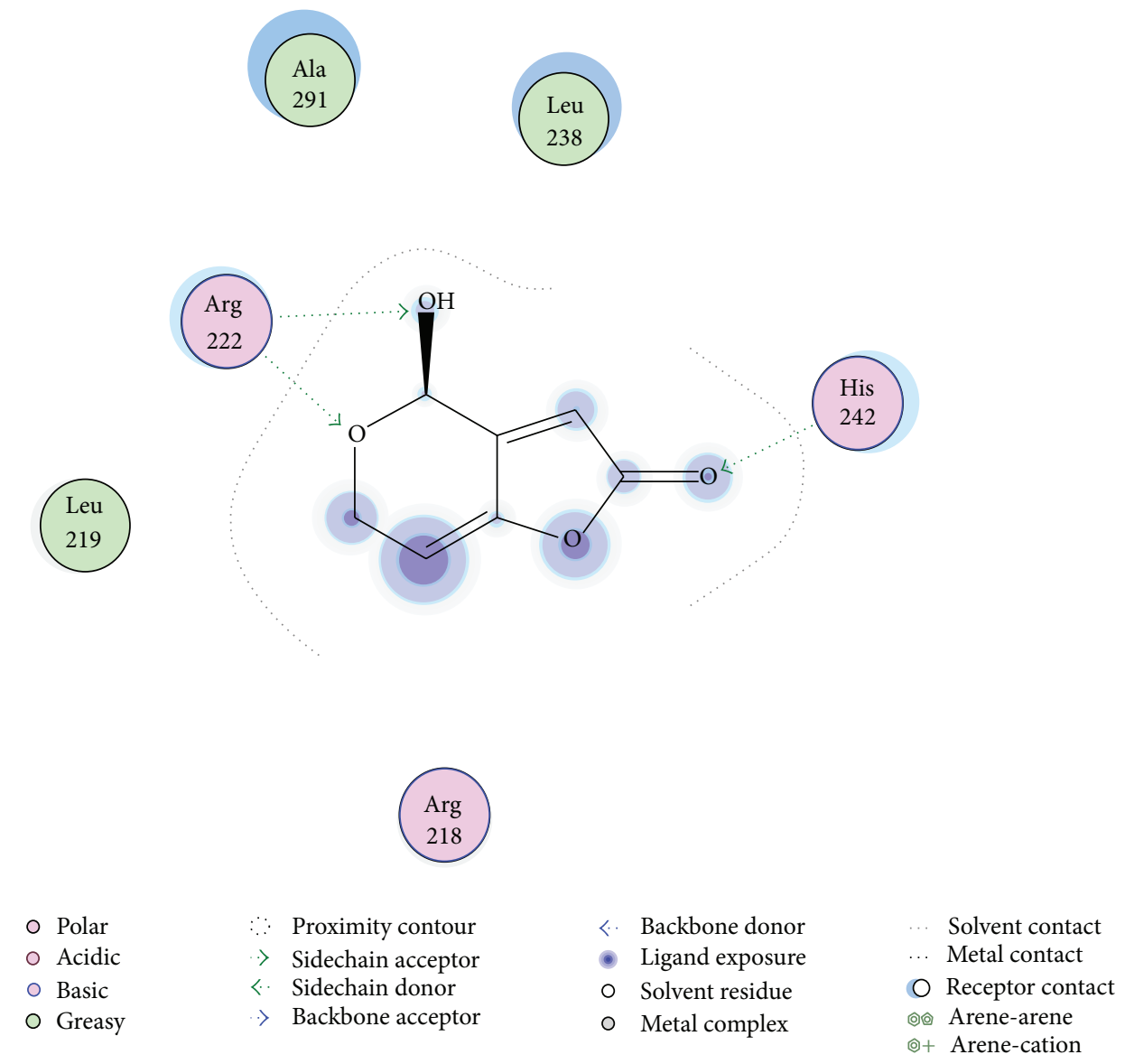

(b)

Figure 8: (a) The interaction mode between patulin and HAS. (b) A projection of $8 \mathrm{a}$. The HSA residues are represented by lines, and the patulin structure is represented by a ball-and-stick model. Hydrogen bonds between patulin and HSA are represented by dashed lines. 


\section{Conclusions}

In this study, the interactions of patulin with HSA were investigated under simulated physiological conditions using different spectroscopic methods: AFM and molecular modeling. Steady-state and time-resolved fluorescence spectra suggest that a dynamic quenching mechanism occurred in patulinHSA complexes. The binding parameters for the reaction were determined using the Stern-Volmer equation. The thermodynamic parameters obtained at different temperatures and the molecular modeling results indicate that hydrophobic interaction was the dominant binding force and also suggest the formation of hydrogen bonds between patulin and HSA. This finding is expected to elucidate the toxigenicity of patulin when it is combined with the biomolecular function effect, transmembrane transport, toxicological testing, and other experiments.

\begin{tabular}{|c|c|}
\hline \multicolumn{2}{|c|}{ Abbreviations } \\
\hline PMTD & The provisional maximum \\
\hline NOEL: & A no-observed-effect level \\
\hline HSA: & Human serum albumin \\
\hline CD: & Circular dichroism \\
\hline AFM: & Atomic force microscopy \\
\hline Trp: & Tryptophan \\
\hline Tyr: & Tyrosine \\
\hline Phe: & Phenylalanine. \\
\hline
\end{tabular}

\section{Conflict of Interests}

The authors declare that they have no conflict of interests.

\section{Authors' Contribution}

Li Yuqin conceived and designed the study, carried out atomic force microscopy and data analysis, interpreted the entire results, and drafted the paper. You Guirong carried out steady-state fluorescence spectra and data analysis. Yang Zhen carried out UV-Vis spectroscopy and helped to draft the paper. Liu Caihong carried out time-resolved fluorescence spectra and data analysis. Jia Baoxiu carried out circular dichroism spectra and data analysis. Chen Jiao carried out molecular modeling and data analysis. Guo Yurong participated in the design of the study and interpreted the results. All authors read and approved the final paper.

\section{Acknowledgment}

This work was supported by the China Agriculture Research System (Cars-28).

\section{References}

[1] O. Puel, P. Galtier, and I. P. Oswald, "Biosynthesis and toxicological effects of patulin,” Toxins, vol. 2, no. 4, pp. 613-631, 2010.

[2] H. M. Gashlan, "High performance liquid chromatographic determination of patulin in apple juice: investigation of its contamination levels in Saudi Arabia," Scientific Research and Essays, vol. 4, no. 2, pp. 69-72, 2009.

[3] K. Baert, F. Devlieghere, H. Flyps et al., "Influence of storage conditions of apples on growth and patulin production by Penicillium expansum," International Journal of Food Microbiology, vol. 119, no. 3, pp. 170-181, 2007.

[4] M. O. Moss, "Fungi, quality and safety issues in fresh fruits and vegetables," Journal of Applied Microbiology, vol. 104, no. 5, pp. 1239-1243, 2008.

[5] H. Morales, S. Marín, X. Centelles, A. J. Ramos, and V. Sanchis, "Cold and ambient deck storage prior to processing as a critical control point for patulin accumulation," International Journal of Food Microbiology, vol. 116, no. 2, pp. 260-265, 2007.

[6] JECFA, Joint FAO/WHO Expert Committee on Food Additives Contaminants, WHO Food Additives Series, Toxicological Evaluation of Certain Food Additives and Contaminants, 1966.

[7] FAO-WHO, Evalution of Certain Food Additives and Contaminants, vol. 896 of World Health Organization Technical Report Series, 2000.

[8] U. Galvalisi, S. Lupo, J. Piccini, and L. Bettucci, "Penicillium species present in Uruguayan salami," Revista Argentina de Microbiologia, vol. 44, no. 1, pp. 36-42, 2012.

[9] C. Behm, W. Föllmann, and G. H. Degen, "Cytotoxic potency of mycotoxins in cultures of v79 lung fibroblast cells," Journal of Toxicology and Environmental Health A: Current Issues, vol. 75, no. 19-20, pp. 1226-1231, 2012.

[10] R. E. Dailey, A. M. Blaschka, and E. A. Brouwer, "Absorption, distribution, and excretion of $\left[{ }^{14} \mathrm{C}\right]$ patulin by rats," Journal of Toxicology and Environmental Health, vol. 3, no. 3, pp. 479-489, 1977.

[11] J. L. Richard, "Some major mycotoxins and their mycotoxicosesan overview," International Journal of Food Microbiology, vol. 119, no. 1-2, pp. 3-10, 2007.

[12] T.-S. Wu, J.-J. Yang, F.-Y. Yu, and B.-H. Liu, "Evaluation of nephrotoxic effects of mycotoxins, citrinin and patulin, on zebrafish (Danio rerio) embryos," Food and Chemical Toxicology, vol. 50, no. 12, pp. 4398-4404, 2012.

[13] R. Ito, H. Yamazaki, K. Inoue, Y. Yoshimura, M. Kawaguchi, and H. Nakazawa, "Development of liquid chromatographyelectrospray mass spectrometry for the determination of patulin in apple juice: Investigation of its contamination in Japan," Journal of Agricultural and Food Chemistry, vol. 52, no. 25, pp. 7464-7468, 2004.

[14] N. Glaser and H. Stopper, "Patulin: mechanism of genotoxicity," Food and Chemical Toxicology, vol. 50, no. 5, pp. 1796-1801, 2012.

[15] S. K. Abraham, A. Eckhardt, R. G. Oli, and H. Stopper, "Analysis of in vitro chemoprevention of genotoxic damage by phytochemicals, as single agents or as combinations," Mutation Research-Genetic Toxicology and Environmental Mutagenesis, vol. 744, no. 2, pp. 117-124, 2012.

[16] R. P. Sharma, "Immunotoxicity of mycotoxins," Journal of Dairy Science, vol. 76, no. 3, pp. 892-897, 1993.

[17] G. Selmanoglu and E. A. Koçkaya, "Investigation of the effects of patulin on thyroid and testis, and hormone levels in growing male rats," Food and Chemical Toxicology, vol. 42, no. 5, pp. 721727, 2004.

[18] G. Selmanoğlu, "Evaluation of the reproductive toxicity of patulin in growing male rats," Food and Chemical Toxicology, vol. 44, no. 12, pp. 2019-2024, 2006.

[19] A. Divsalar, M. J. Bagheri, A. A. Saboury, H. Mansoori-Torshizi, and M. Amani, "Investigation on the interaction of newly 
designed anticancer Pd(II) complexes with different aliphatic tails and human serum albumin," Journal of Physical Chemistry B, vol. 113, no. 42, pp. 14035-14042, 2009.

[20] D. C. Carter and J. X. Ho, "Structure of serum albumin," Advances in Protein Chemistry, vol. 45, pp. 153-203, 1994.

[21] F. Ge, C. Chen, D. Liu, B. Han, X. Xiong, and S. Zhao, "Study on the interaction between theasinesin and human serum albumin by fluorescence spectroscopy," Journal of Luminescence, vol. 130, no. 1, pp. 168-173, 2010.

[22] Y. Yue, X. Chen, J. Qin, and X. Yao, "A study of the binding of C.I. Direct Yellow 9 to human serum albumin using optical spectroscopy and molecular modeling," Dyes and Pigments, vol. 79, no. 2, pp. 176-182, 2008.

[23] Y. Li, X. J. Yao, J. Jin, X. G. Chen, and Z. D. Hu, "Interaction of rhein with human serum albumin investigation by optical spectroscopic technique and modeling studies," Biochimica et Biophysica Acta, vol. 1774, no. 1, pp. 51-58, 2007.

[24] T. O. Hushcha, A. I. Luik, and Y. N. Naboka, "Conformation changes of albumin in its interaction with physiologically active compounds as studied by quasi-elastic light scattering spectroscopy and ultrasonic method," Talanta, vol. 53, no. 1, pp. 29-34, 2000.

[25] M. Purcell, J. F. Neault, T. Malonga, H. Arakawa, R. Carpentier, and H. A. Tajmir-Riahi, "Interactions of atrazine and 2,4-D with human serum albumin studied by gel and capillary electrophoresis, and FTIR spectroscopy," Biochimica et Biophysica Acta-Protein Structure and Molecular Enzymology, vol. 1548, no. 1, pp. 129-138, 2001.

[26] V. Berger, A. Gabriel, T. Sergent, A. Trouet, Y. Larondelle, and Y. Schneider, "Interaction of ochratoxin A with human intestinal Caco-2 cells: possible implication of a multidrug resistanceassociated protein MRP2," Toxicology Letters, vol. 140-141, pp. 465-476, 2003.

[27] D. Silva, C. M. Cortez, J. Cunha-Bastos, and S. R. W. Louro, "Methyl parathion interaction with human and bovine serum albumin," Toxicology Letters, vol. 147, no. 1, pp. 53-61, 2004.

[28] S. J. Uddin, J. A. Shilpi, G. M. M. Murshid, A. A. Rahman, S. M. Marder, and M. A. Alam, "Determination of the binding sites of arsenic on bovine serum albumin using warfarin site-I specific probe and diazepam site-II specific probe," The Journal of Biological Sciences, vol. 4, no. 5, pp. 609-612, 2004.

[29] F. Ding, W. Liu, J. Diao, and Y. Sun, "Characterization of Alizarin Red S binding sites and structural changes on human serum albumin: A biophysical study," Journal of Hazardous Materials, vol. 186, no. 1, pp. 352-359, 2011.

[30] X. Xie, Z. Wang, X. Zhou, X. Wang, and X. Chen, "Study on the interaction of phthalate esters to human serum albumin by steady-state and time-resolved fluorescence and circular dichroism spectroscopy," Journal of Hazardous Materials, vol. 192, no. 3, pp. 1291-1298, 2011.

[31] Y. Li, H. Wang, B. Jia et al., "Study of the interaction of deoxynivalenol with human serum albumin by spectroscopic technique and molecular modelling," Food Additives \& Contaminants A, vol. 30, no. 2, pp. 356-364, 2013.

[32] Y. Li, B. Jia, H. Wang et al., "The interaction of 2-mercaptobenzimidazole with human serum albumin as determined by spectroscopy, atomic force microscopy and molecular modeling," Colloids and Surfaces B: Biointerfaces, vol. 104, pp. 311-317, 2013.

[33] M. Clark, R. D. Cramer, and N. V. Opdenbosch, "Validation of the general purpose tripos 5.2 forcefield," Journal of Computational Chemistry, vol. 10, no. 8, pp. 982-1012, 1989.
[34] D. Pandit, A. Fiorentino, S. Bindra, and C. A. Venanzi, "Singular value decomposition analysis of the torsional angles of dopamine reuptake inhibitor GBR 12909 analogs: Effect of force field and charges," Journal of Molecular Modeling, vol. 17, no. 6, pp. 1343-1351, 2011.

[35] J. N. Miller, "Recent advances in molecular luminescence analysis," Proceedings of the Analytical Division of the Chemical Society, vol. 16, no. 3, pp. 203-208, 1979.

[36] H. M. Zhang, T. T. Chen, Q. H. Zhou, and Y. Q. Wang, "Binding of caffeine, theophylline, and theobromine with human serum albumin: a spectroscopic study," Journal of Molecular Structure, vol. 938, no. 1-3, pp. 221-228, 2009.

[37] J. R. Lakowicz, Principles of Fluorescence Spectroscopy, Springer, New York, NY, USA, 3rd edition, 2006.

[38] A. V. Pastukhov, L. A. Levchenko, and A. P. Sadkov, "Spectroscopic study on binding of rutin to human serum albumin," Journal of Molecular Structure, vol. 842, no. 1-3, pp. 60-66, 2007.

[39] M. K. Helms, C. E. Petersen, N. V. Bhagavan, and D. M. Jameson, "Time-resolved fluorescence studies on site-directed mutants of human serum albumin," FEBS Letters, vol. 408, no. 1, pp. 67-70, 1997.

[40] X. M. He and D. C. Carter, "Atomic structure and chemistry of human serum albumin," Nature, vol. 358, no. 6383, pp. 209-215, 1992.

[41] S. N. Timasheff, “Thermodynamics of protein interactions," in Proteins of Biological Fluids, H. Peeters, Ed., p. 511, Pergamon, Oxford, UK, 1972.

[42] P. D. Ross and S. Subramanian, "Thermodynamics of protein association reactions: forces contributing to stability," Biochemistry, vol. 20, no. 11, pp. 3096-3102, 1981.

[43] D. Kowalczyk, J.-. Marsault, and S. Slomkowski, "Analysis of in vitro chemoprevention of genotoxic damage by phytochemicals, as single agents or as combinations," Mutation ResearchGenetic Toxicology and Environmental Mutagenesis, vol. 274, no. 6, pp. 513-519, 1996.

[44] G. Sudlow, D. J. Birkett, and D. N. Wade, "The characterization of two specific drug binding sites on human serum albumin," Molecular Pharmacology, vol. 11, no. 6, pp. 824-832, 1975.

[45] G. Sudlow, D. J. Birkett, and D. N. Wade, "Further characterization of specific drug binding sites on human serum albumin," Molecular Pharmacology, vol. 12, no. 6, pp. 1052-1061, 1976. 

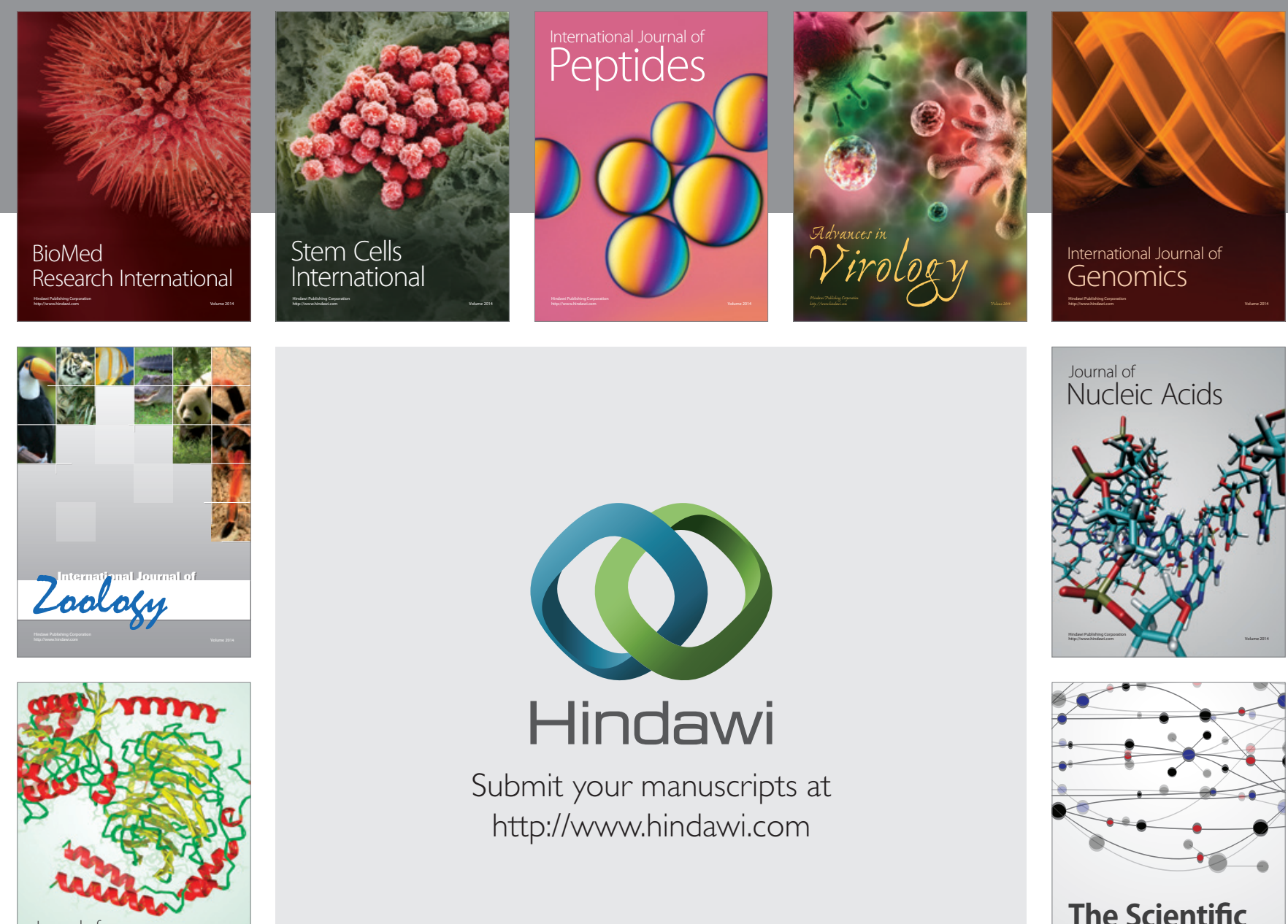

Submit your manuscripts at

http://www.hindawi.com

Journal of
Signal Transduction
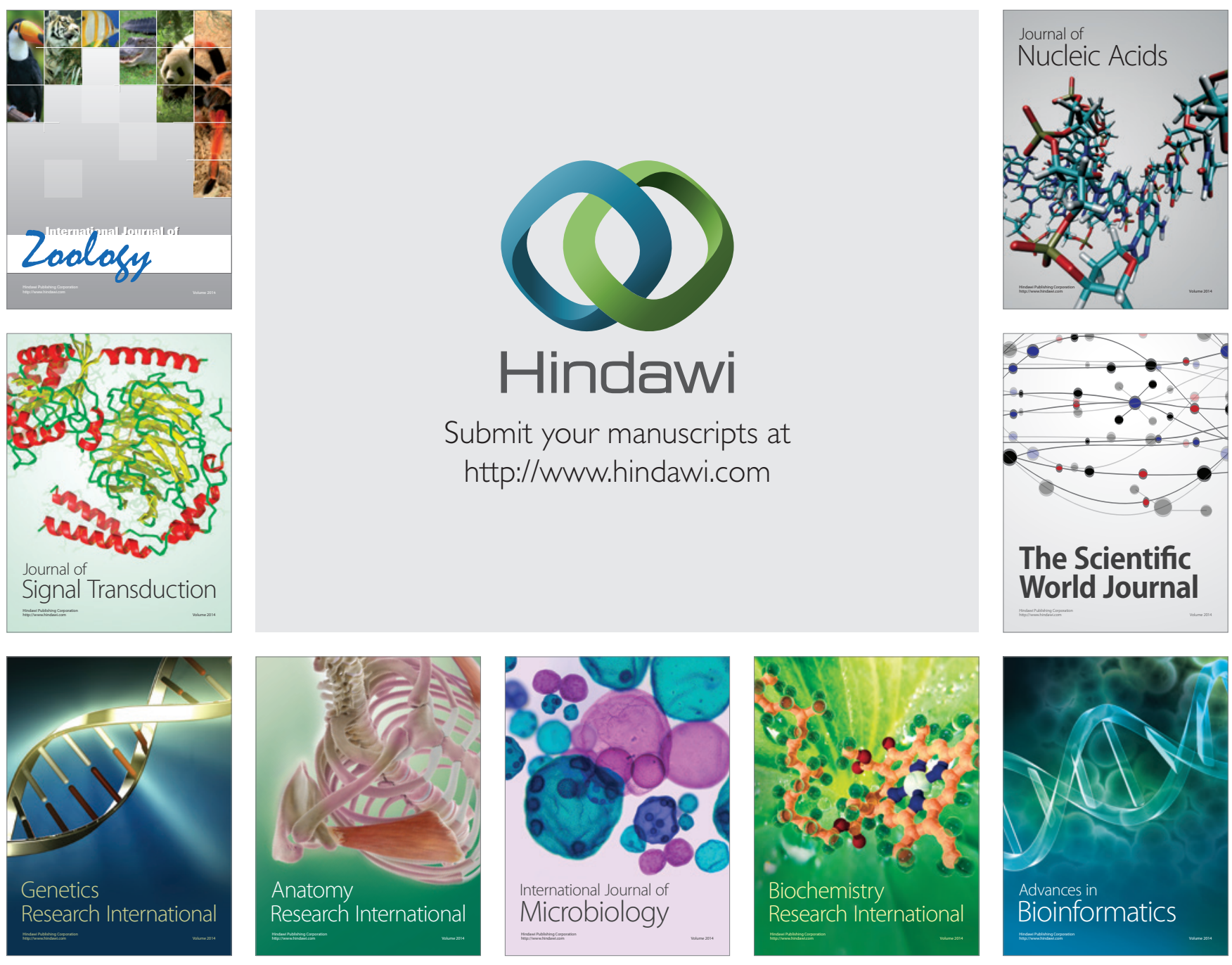

The Scientific World Journal
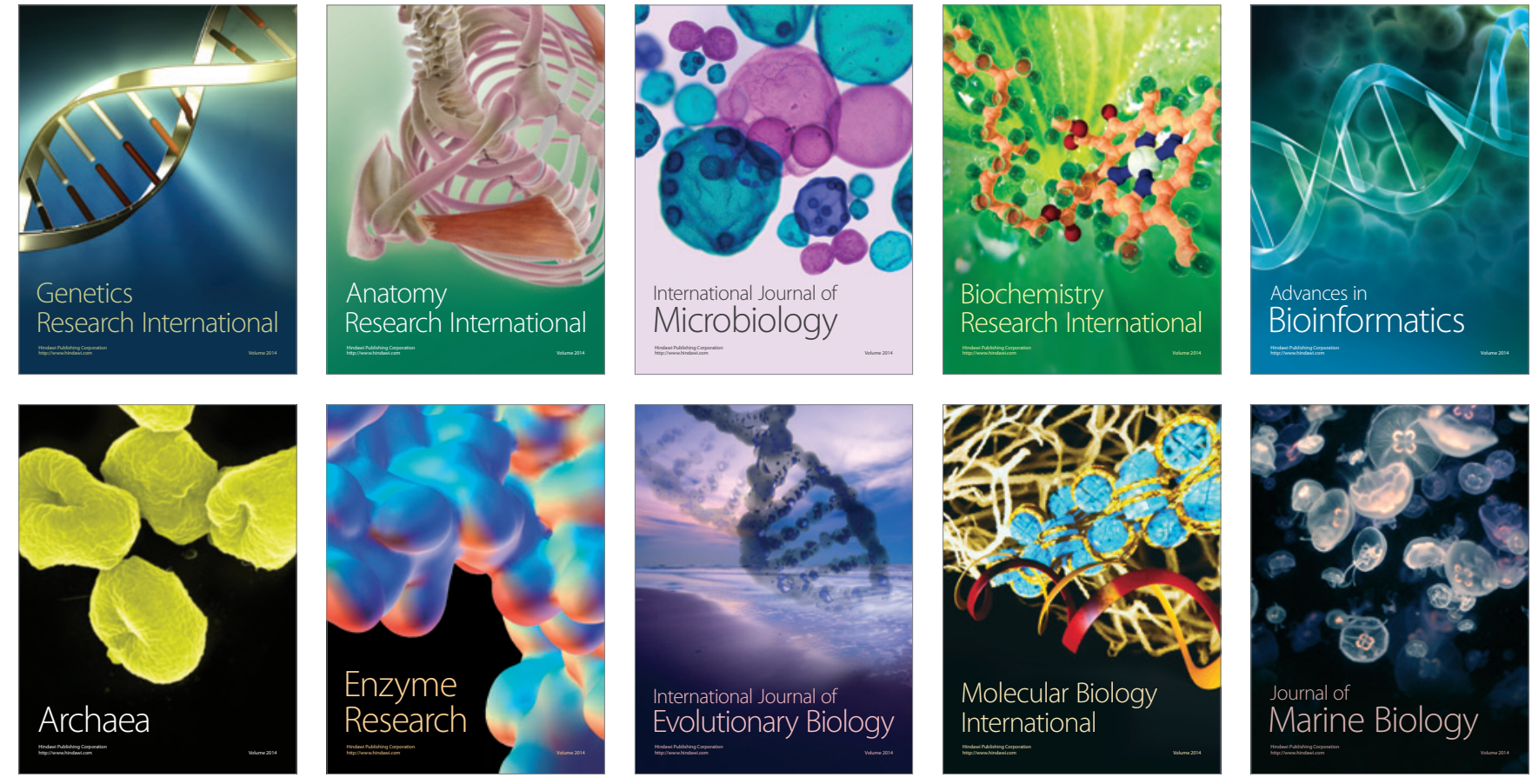